\title{
Narrow-band UVB radiation promotes dendrite formation by activating Rac1 in B16 melanoma cells
}

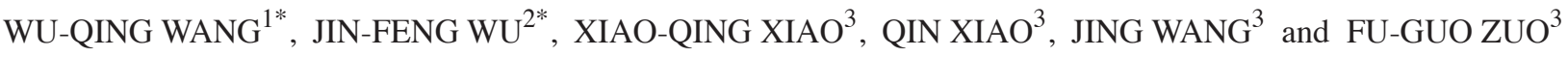 \\ ${ }^{1}$ Department of Dermatology, The Central Hospital of Minhang, Shanghai 201199; ${ }^{2}$ Department of Dermatology, \\ Huashan Hospital, Fudan University, Shanghai 200040; ${ }^{3}$ Department of Dermatology, East Hospital, \\ School of Medicine, Tongji University, Shanghai 200120, P.R. China
}

Received April 12,2013; Accepted June 27, 2013

DOI: $10.3892 / \mathrm{mco} .2013 .145$

\begin{abstract}
Melanocytes are found scattered throughout the basal layer of the epidermis. Following hormone or ultraviolet (UV) light stimulation, the melanin pigments contained in melanocytes are transferred through the dendrites to the surrounding keratinocytes to protect against UV light damage or carcinogenesis. This has been considered as a morphological indicator of melanocytes and melanoma cells. Small GTPases of the Rho family have been implicated in the regulation of actin reorganization underlying dendrite formation in melanocytes and melanoma cells. It has been proven that ultraviolet light plays a pivotal role in melanocyte dendrite formation; however, the molecular mechanism underlying this process has not been fully elucidated. The effect of small GTPases, such as Racl and RhoA, on the morphology of B16 melanoma cells treated with narrow-band UVB radiation was investigated. The morphological changes were observed under a phase contrast microscope and the F-actin microfilament of the cytoskeleton was observed under a laser scanning confocal microscope. The pull-down assay was performed to detect the activity of the small GTPases Racl and RhoA. The morphological changes were evident, with globular cell bodies and increased numbers of tree branch-like dendrites. The cytoskeletal F-actin appeared disassembled following narrow-band UVB irradiation of B16 melanoma cells. Treatment of B16 melanoma cells with narrow-band UVB radiation resulted in the activation of Rac1 in a time-dependent manner. In conclusion, the present study may provide a novel method through which narrow-band UVB radiation may be used to promote dendrite formation by
\end{abstract}

Correspondence to: Dr Fu-Guo Zuo, Department of Dermatology, East Hospital, School of Medicine, Tongji University, 150 Jimo Road, Shanghai 200120, P.R. China

E-mail: zfg5747@126.com

*Contributed equally

Key words: narrow-band ultraviolet B radiation, Rac1, F-actin rearrangement, dendricity, B16 melanoma cells activating the Rac1 signaling pathway, resulting in F-actin rearrangement in B16 melanoma cells.

\section{Introduction}

Melanocytes are derived from the neural crest and are found scattered throughout the basal layer of the epidermis. In response to hormones or ultraviolet (UV) light, the melanin pigments contained in melanocytes are transferred to the surrounding keratinocytes through the tips of the dendrites to protect against UV light damage or carcinogenesis. This has been considered a morphological indicator of melanocytes and melanoma cells. During this process, dendrite extension is critical, since melanocytes constitute a minor population in the epidermis (1-3). Previous studies demonstrated that the dendritic outgrowth of melanocytes and melanoma cells is promoted by UV light irradiation, growth factors and cAMP-elevating agents (4-6).

The rearrangement of the actin cytoskeleton promoting dendritic outgrowth and the underlying signaling mechanisms in melanocytes and melanoma cells were previously investigated $(3,4,7)$. The identification of the small GTPases of the Rho family has elucidated the molecular signaling underlying cell shape alterations. The small GTPases of the Rho family regulate several intracellular processes, including cytoskeletal reorganization, cell motility, cell-cell adhesion and apoptosis (8-11), as well as regulation of the cell cycle, oncogenesis and gene transcription $(12,13)$. Twenty genes encoding proteins belonging to the Rho family of small GTPases have been described in humans thus far. With regards to cell morphology, three of the small GTPases appear to be more important: Rac1, RhoA and Cdc42 (14). The role of Rac1 and RhoA in dendrite formation by human melanocytes and melanoma cells was defined by several studies. Overexpression of the constitutive active form of MKK6 resulted in significant elongation of the dendrites via the upregulation of $\mathrm{Cdc} 42$ and Racl expression in the SK-mel-24 human melanoma cell line (15). A study conducted by Ito et al (16) demonstrated that centaureidin may act via the Rho signaling pathway to inhibit dendrite outgrowth in melanocytes. Furthermore, Scott and Leopardi (17) reported that cAMP-mediated dendrite formation in B16 melanoma cells occurs via the upregulation of Rac and inhibition of Rho activity. 
There is substantial evidence suggesting that UV irradiation plays a pivotal role in regulating melanocyte dendricity. In the development of melasma, the strongest predictors are UV light exposure and genetic factors $(18,19)$. Furthermore, the histological analysis of melasma lesions revealed that epidermal melanocytes exhibited a strong staining intensity and more dendrites (20). Reflectance confocal imaging of murine skin in vivo following exposure to UVB light highlighted the dendricity of melanocytes (3). Although it is likely that UV light is a primary stimulus for melanocyte dendrite formation, the possible molecular mechanisms have not been fully elucidated. The effect of narrow-band UVB radiation on the morphological changes and the actin cytoskeleton in B16 melanoma cells was investigated, with the aim of elucidating the mechanism underlying this process.

\section{Materials and methods}

Cell culture and narrow-band UVB radiation treatment. B16 melanoma cells are transformed melanocytes and suitable as a physiological model of normal melanocytes. In the present study, B16 melanoma cells were investigated to elucidate the mechanism of dendrite formation induced by narrow-band UVB radiation. B16 melanoma cells (Institute of Biochemistry and Cell Biology, Shanghai Institutes for Biological Sciences, Chinese Academy of Sciences, Shanghai, China) were maintained in RPMI-1640 medium (Gibco, Carlsbad, CA, USA) supplemented with $10 \%$ fetal bovine serum (FBS) and antibiotics (100 IU/ml penicillin and $50 \mathrm{mg} / \mathrm{ml}$ streptomycin) (Invitrogen, Grand Island, NY, USA) in a humidified atmosphere of $5 \% \mathrm{CO}_{2}$ at $37^{\circ} \mathrm{C}$. The cells were exposed to various doses of radiation by a specific UVB lamp emitting a peak wavelength of $311 \mathrm{~nm}$ with an intensity of $1 \mathrm{~mW} / \mathrm{cm}^{2}$ (Philips, Amsterdam, Netherlands). During irradiation, the medium was replaced with phosphate-buffered saline (PBS) to avoid the formation of medium-derived toxic photoproducts induced by UVB exposure. Immediately after phototreatment, PBS was removed and media were added to the cells. The subsequent experiments were performed three times in triplicate.

Cell viability assay. The cells were seeded in 96-well plates at a density of $1 \times 10^{4}$ cells/well and incubated for $24 \mathrm{~h}$. Subsequently, the cells were exposed to various doses of narrow-band UVB radiation $\left(0,25,50,100,200\right.$ and $\left.300 \mathrm{~mJ} / \mathrm{cm}^{2}\right)$ and were incubated for an additional $24 \mathrm{~h}$. Cell viability was assessed using a commercially available kit (Cell Counting kit-8; Dojindo Co., Ltd., Kumamoto, Japan) according to the manufacturer's instructions to determine the appropriate dose of narrow-band UVB radiation for the subsequent experiments. The colorimetric absorbance was recorded at $490 \mathrm{~nm}$ using the ELx800 microplate reader (BioTek, Winooski, VT, USA).

Dendrite formation assay. B16 melanoma cells (1x10 ${ }^{5}$ cells/well) growing in 6-well microplates were cultured for $24 \mathrm{~h}$ and were subsequently irradiated with narrow-band UVB light $\left(100 \mathrm{~mJ} / \mathrm{cm}^{2}\right)$. Following incubation for a further $24 \mathrm{~h}$ in an FBS-free medium (in order to avoid the effect of growth factors in FBS on dendrite formation), the morphological changes were observed under an ELWD 0.3 phase contrast microscope (Nikon, Tokyo, Japan). The number of dendrites per cell was determined for $\sim 100$ cells from each experiment.

Immunofluorescence microscopy. B16 melanoma cells growing on coverslips were placed within culture plates $\left(1 \times 10^{5}\right.$ cells), cultured for $24 \mathrm{~h}$ and then irradiated with narrow-band UVB light $\left(100 \mathrm{~mJ} / \mathrm{cm}^{2}\right)$. Following incubation for an additional $24 \mathrm{~h}$ in FBS-free medium for immunofluorescence studies, the B16 melanoma cells growing on glass coverslips were briefly rinsed with PBS and fixed in 4\% formaldehyde, then permeabilized with $0.5 \%$ Triton X-100 (Sigma-Aldrich, St. Louis, MO, USA) for $2 \mathrm{~min}$ for detection of cytoskeletal F-actin. The cells were washed for $10 \mathrm{~min}$ in PBS and exposed to rhodamine phalloidin staining (Cytoskeleton Inc., Denver, CO, USA) diluted in PBS containing $1 \%$ of BSA, applied for $30 \mathrm{~min}$ at $37^{\circ} \mathrm{C}$. The coverslips were rinsed with PBS for 10 min and observed and photographed with a Leica TCS SP2 laser scanning confocal microscope (LSCM) (Leica, Solms, Germany).

Pull-down assay. An equal number of B16 melanoma cells $\left(\sim 1 \times 10^{6}\right.$ cells) was cultured, followed by lysis in ice-cold cell lysis buffer supplemented with $1 \mathrm{X}$ protease inhibitor cocktail after narrow-band UVB irradiation for the indicated time. The pull-down assay was implemented for the detection of GTP-Rac1 and -RhoA using a commercially available kit (Cytoskeleton Inc.) according to the manufacturer's protocol. In brief, a pre-determined amount of PAK-PBD beads $(20 \mu \mathrm{g})$ or GST-Rhotekin-RBD beads $(10 \mu \mathrm{g})$ was added to the cleared lysates and incubated at $4^{\circ} \mathrm{C}$ on a rotator for $1 \mathrm{~h}$. GTP-bound proteins were captured onto the beads and pelleted. The beads were rinsed with a wash buffer and GTP-bound protein was eluted with $2 \mathrm{X}$ Laemmli sample buffer. Samples were immunoblotted after $12.5 \%$ SDS-PAGE using standard procedures. To assess loading equality among different lysates, a portion of each lysate was removed prior to the addition of PAK-PBD beads or GST-Rhotekin-RBD beads and blotted with antiRhoA and anti-Rac1 antibodies. The expression of GTP-Rac1 and GTP-RhoA was detected using the Plus-ECL method. Densitrometric analysis was performed using LAS-3000 ECL image analysis system (Fujifilm Medical Systems, Tokyo, Japan).

\section{Results}

Effects of narrow-band UVB radiation on B16 melanoma cell viability. B16 melanoma cells were irradiated with different doses of narrow-band UVB light. The results demonstrated that irradiation with narrow-band UVB light at 25, 50 and $100 \mathrm{~mJ} / \mathrm{cm}^{2}$ exerted no stimulatory effect on cell proliferation compared to control groups. However, 200 and $300 \mathrm{~mJ} / \mathrm{cm}^{2}$ of irradiation significantly reduced the cell survival rate. According to these results, narrow-band UVB irradiation at $100 \mathrm{~mJ} / \mathrm{cm}^{2}$ was selected as the appropriate dose for the subsequent experiments.

Morphological changes in B16 melanoma cells following narrow-band $U V B$ irradiation. To investigate the effects of narrow-band UVB radiation on dendrite formation, B16 melanoma cells were irradiated with $100 \mathrm{~mJ} / \mathrm{cm}^{2}$ 
A

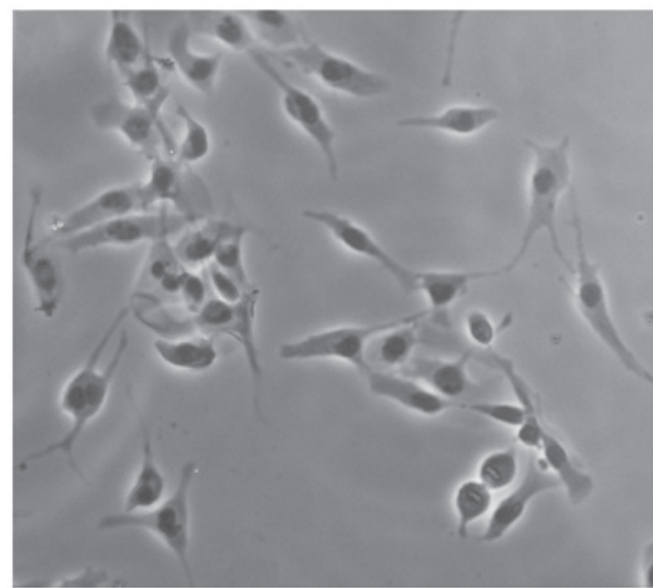

B

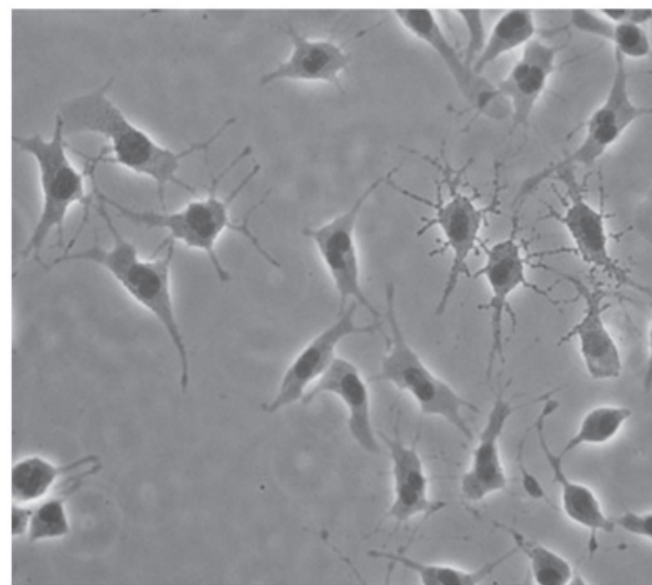

Figure 1. Morphological changes in B16 melanoma cells following narrow-band UVB irradiation. The morphological changes were observed under a phase contrast microscope (bar, $50 \mu \mathrm{M}$ ). (A) Non-irradiated B16 cells exhibit 2-3 dendrites. (B) B16 melanoma cells with globular cell bodies and an increased number of tree branch-like dendrites at $24 \mathrm{~h}$ following narrow-band UVB irradiation $\left(100 \mathrm{~mJ} / \mathrm{cm}^{2}\right)$.
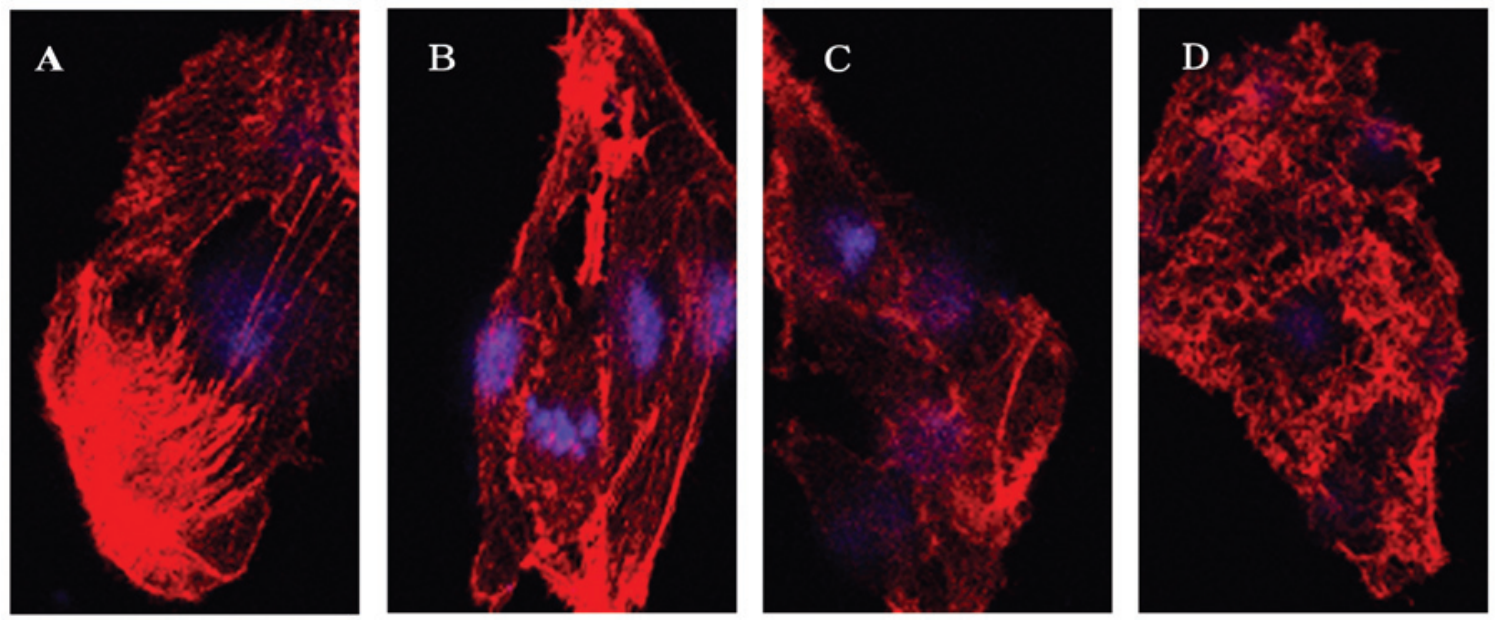

Figure 2. Effects of narrow-band UVB radiation on cytoskeletal F-actin in B16 melanoma cells. (A) Laser scanning confocal microscopy (LSCM) revealed that F-actin appeared organized in numerous clear stress fibers crossing the cytoplasm in non-irradiated cells. Following narrow-band UVB irradiation $\left(100 \mathrm{~mJ} / \mathrm{cm}^{2}\right)$, these stress fibers became obscure as F-actin was disassembled. This event could be observed as early as (B) 30 min, becoming more evident at (C) $60 \mathrm{~min}$, with the appearance of punctate spots at (D) $6 \mathrm{~h}$.

narrow-band UVB light for $24 \mathrm{~h}$. The dendrite number in 100 B16 melanoma cells from each experiment was manually counted. The morphological changes in B16 melanoma cells induced by narrow-band UVB radiation were evident, with markedly globular cell bodies and significantly increased numbers of tree branch-like dendrites $(5.52 \pm 1.35 /$ per cell) compared to untreated cells, which exhibited 2-3 dendrites $(2.39 \pm 0.36 /$ per cell) (Fig. 1).

Effects of narrow-band UVB radiation on cytoskeletal $F$-actin in B16 melanoma cells. The changes in the cytoskeletal F-actin were observed using an LSCM. F-actin appeared to be organized in numerous clear stress fibers crossing the cytoplasm in non-irradiated cells (Fig. 2A). However, these stress fibers became obscure as actin was disassembled following narrow-band UVB irradiation $\left(100 \mathrm{~mJ} / \mathrm{cm}^{2}\right)$ in a time-dependent manner. This event was observed as early as 30 min (Fig. 2B) after irradiation and became more evident with the appearance of punctate spots at $6 \mathrm{~h}$ (Fig. 2C and D).
Effects of narrow-band UVB radiation on the activity of GTP-Racl and-RhoA in B16 melanoma cells. The pull-down analysis (Fig. 3A) revealed that GTP-Rac1 protein expression was significantly increased at $15 \mathrm{~min}$ and reached twice the baseline levels at $30 \mathrm{~min}$ after narrow-band UVB irradiation $\left(100 \mathrm{~mJ} / \mathrm{cm}^{2}\right)$, followed by a minor decrease, although the protein levels remained elevated at 60 and 120 min compared to the control cells. The GTP-RhoA levels were marginally altered within the first $30 \mathrm{~min}$ after narrow-band UVB irradiation $\left(100 \mathrm{~mJ} / \mathrm{cm}^{2}\right)$, were elevated at $60 \mathrm{~min}$ and by 1.6 -fold at 120 min compared to the control cells (Fig. 3B).

\section{Discussion}

Over the last few years, the molecular mechanisms responsible for melanocyte dendricity have attracted increasing attention in the field of melanocyte research. By examining the cytoskeletal components during cAMP-induced dentricity in B16F10 cells, it was observed that the 

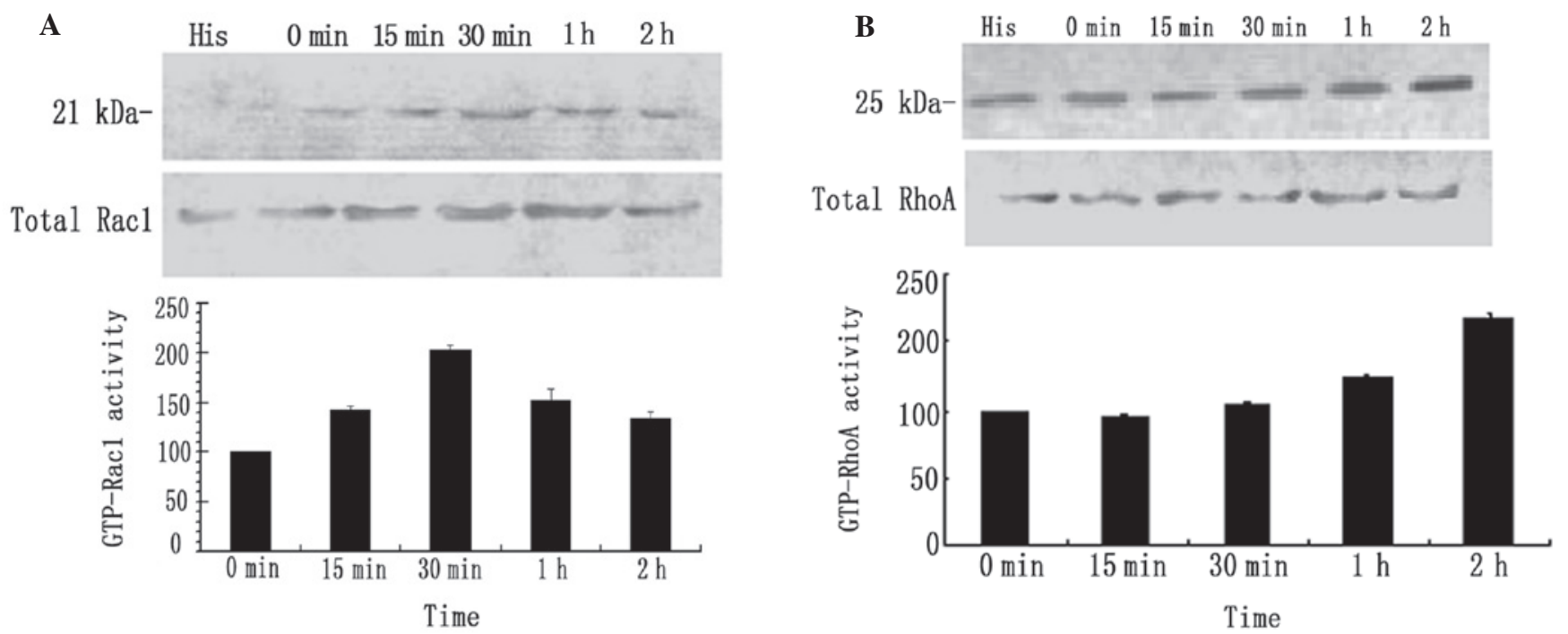

Figure 3. Effects of narrow-band UVB radiation on the activity of GTP-RhoA and -Rac1 in B16 melanoma cells. (A) The pull-down analysis revealed that the GTP-Rac1 protein expression was significantly increased at $15 \mathrm{~min}$ and by 2 -fold at $30 \mathrm{~min}$ following narrow-band UVB irradiation (100 $\left.\mathrm{mJ} / \mathrm{cm}^{2}\right)$, followed by a minor decrease, although the protein levels remained elevated at 60 and 120 min compared to control cells. (B) GTP-RhoA levels were marginally changed during the first $30 \mathrm{~min}$ after irradiation, were elevated at $60 \mathrm{~min}$ and by 1.6 -fold at $120 \mathrm{~min}$.

cAMP-dependent dendricity was accompanied by a significant reorganization of the actin cytoskeleton (21). It has been verified previously that UV light irradiation is involved in the regulation of dendricity in human or mice melanocytes, as described above $(3,18-20)$.

In the present study, the effect of narrow-band UVB radiation on morphological changes in B16 melanoma cells in vitro was observed. Marked morphological changes were evident in B16 melanoma cells treated with $100 \mathrm{~mJ} / \mathrm{cm}^{2}$ narrow-band UVB radiation, with globular cell bodies and increased numbers of tree branch-like dendrites as compared to untreated cells, which merely exhibited 2-3 dendrites. Furthermore, the changes in the cytoskeletal F-actin microfilament in response to narrow-band UVB radiation were investigated using LSCM. LSCM revealed that F-actin appeared organized in numerous clear stress fibers crossing the cytoplasm in non-irradiated cells. However, these stress fibers became obscure as actin was disassembled following narrow-band UVB irradiation in a time-dependent manner. This event was observed as early as 30 min following irradiation and became more evident with the appearance of punctate spots at $6 \mathrm{~h}$. These results suggest that narrow-band UVB radiation may promote the disorganization of cytoskeletal F-actin, leading to morphological changes such as globular cell bodies and increased numbers of dendrites in B16 melanoma cells.

The small GTPases, including RhoA, Rac1 and Cdc42, have been shown to regulate the assembly and disassembly of the actin cytoskeleton in neural crest-derived cells $(3,21)$. Another possible explanation for the morphological changes exhibited by B16 melanoma cells exposed to narrow-band UVB radiation is the up- or downregulation of these small GTPases, which is based on the findings of previous studies, according to which Rac1 activation and RhoA inhibition induce elongation of the dendrites and the activated RhoA stimulates dendrite retraction (5,22-24).

In this study, we investigated the effect of narrow-band UVB radiation on Racl and RhoA activity using the pull-down assay. The analysis revealed that GTP-Rac1 protein expression was significantly increased at $15 \mathrm{~min}$ and had doubled at 30 min following narrow-band UVB irradiation, which was followed by a minor decrease, although the protein levels remained elevated at 1 and $2 \mathrm{~h}$ compared to non-irradiated cells. GTP-RhoA expression exhibited minor alterations in the first 30 min following narrow-band UVB irradiation, was elevated at $1 \mathrm{~h}$ and by 1.6 -fold at $2 \mathrm{~h}$ compared to control cells. Unlike the overexpression of Rac1, RhoA activity in B16 melanoma cells exhibited no significant change in the short period after narrow-band UVB irradiation, although a delayed elevation was observed. The reason for this finding has not been elucidated. Ridley et al (25) concluded that a linear hierarchy exists, with Rac activating Rho, which appears to be consistent with the results of our study. It was hypothesized that Rac-dependent Rho activation in response to narrow-band UVB irradiation may serve as a negative feedback to avoid exorbitant dendrite extension promoted by the activated Rac1, according to the role of RhoA activation in dendrite retraction in B16 melanoma cells (23). In summary, our results suggest that narrow-band UVB radiation specifically activates the Rac1 signaling pathway, leading to F-actin rearrangement and resulting in increased dendrite formation in B16 melanoma cells.

\section{Acknowledgements}

The authors would like to thank Wang Li and Wang Lei (Department of Neurobiology, Fudan University Medical School) for performing the laser scanning confocal microscope test and Zhang Guo-Ping (Institute of Biomedical Research, Fudan University) for providing assistance in the laboratory.

\section{References}

1. Chakraborty AK, Funasaka Y, Araki K, Horikawa T and Ichihashi M: Evidence that the small GTPase Rab8 is involved in melanosome traffic and dendrite extension in B16 melanoma cells. Cell Tissue Res 314: 381-388, 2003. 
2. Fitzpatrick B, Miyamoto M and Ishikawa K: The evolution of concepts of melanin biology. Arch Dermatol 96: 305-323, 1967.

3. Scott G: Rac and Rho: the story behind melanocyte dendrite formation. Pigment Cell Res 15: 322-330, 2002.

4. Hara M, Yaar M and Gilchrest BA: Endothelin-1 of keratinocyte origin is a mediator of melanocyte dendricity. J Invest Dermatol 105: 744-748, 1995.

5. Busca R, Bertolotto C, Abbe P, Engalo W, Ishizaki T, Narumiya S, Boquet P, Ortonne JP and Ballotti R: Inhibition of Rho is required for cAMP-induced melanoma cell differentiation. Mol Biol Cell 9: 1367-1378, 1998.

6. Yoshida M, Takahashi Y and Inoue S: Histamine induces melanogenesis and morphologic changes by protein kinase A activation via $\mathrm{H} 2$ receptors in human normal melanocytes. J Invest Dermatol 114: 334-342, 2000.

7. Hata K, Hori K, Murata J and Takahashi S: Remodeling of actin cytoskeleton in lupeol-induced B16 2F2 cell differentiation. J Biochem 138: 467-472, 2005.

8. Nowak JM, Grzanka A,Zuryń A and Stepień A: The Rho protein family and its role in the cellular cytoskeleton. Postepy Hig Med Dosw (Online) 62: 110-117, 2008 (In Polish).

9. Parri M and Chiarugi P: Rac and Rho GTPases in cancer cell motility control. Cell Commun Signal 8: 23, 2010.

10. Haass NK, Smalley KS, Li L and Herlyn M: Adhesion, migration and communication in melanocytes and melanoma. Pigment Cell Res 18: 150-159, 2005.

11. Ongusaha PP, Kim HG, Boswell SA, Ridley AJ, Der CJ, Dotto GP Kim YB, Aaronson SA and Lee SW: RhoE is a pro-survival p53 target gene that inhibits ROCK I-mediated apoptosis in response to genotoxic stress. Curr Biol 16: 2466-2472, 2006.

12. Benitah SA, Valerón PF and Lacal JC: ROCK and nuclear factor- $\kappa \mathrm{B}$-dependent activation of cyclooxygenase-2 by Rho GTPases: effects on tumor growth and therapeutic consequences. Mol Biol Cell 14: 3041-3054, 2003.

13. Etienne-Manneville S and Hall A: Rho GTPases in cell biology. Nature 420: 629-635, 2002.

14. Burridge $\mathrm{K}$ and Wennerberg $\mathrm{K}$ : Rho and Rac take center stage. Cell 116: 167-179, 2004
15. Kim MY, Choi TY, Kim JH, Lee JH, Kim JG, Sohn KC, Yoon KS, Kim CD, Lee JH and Yoon TJ: MKK6 increases the melanocyte dendricity through the regulation of Rho family GTPases. J Dermatol Sci 60: 114-119, 2010

16. Ito Y, Kanamaru A and Tada A: Centaureidin promotes dendrite retraction of melanocytes by activating Rho. Biochim Biophys Acta 1760: 487-494, 2006.

17. Scott G and Leopardi S: The cAMP signaling pathway has opposing effects on Rac and Rho in B16F10 cells: implications for dendrite formation in melanocytic cells. Pigment Cell Res 16: 139-148, 2003

18. Jimbow $\mathrm{M}$ and Jimbow K: Pigmentary disorders in oriental skin. Clin Dermatol 7: 11-27, 1989.

19. Pichardo R, Vallejos Q, Feldman SR, Schulz MR, Verma A, Quandt SA and Arcury TA: The prevalence of melasma and its association with quality of life in adult male Latino migrant workers. Int J Dermatol 48: 22-26, 2009.

20. Kang WH, Yoon KH, Lee ES, Kim J, Lee KB, Yim H, Sohn S and Im S: Melasma: histopathological characteristics in 56 Korean patients. Br J Dermatol 146: 228-237, 2002.

21. Hall A: Rho GTPases and the control of cell behaviour. Biochem Soc Trans 33 (Pt 5): 891-895, 2005.

22. Scott GA and Cassidy L: Rac1 mediates dendrite formation in response to melanocyte stimulating hormone and ultraviolet light in a murine melanoma model. J Invest Dermatol 111: 243-250, 1998.

23. Katoh H, Aoki J, Ichikawa A and Negishi M: p160 RhoA-binding kinase induces neurite retraction. J Biol Chem 273: 2489-2492, 1998.

24. Sander EE, ten Klooster JP, van Delft S, van der Kammen RA and Collard JG: Rac downregulates Rho activity: reciprocal balance between both GTPases determines cellular morphology and migratory behavior. J Cell Biol 147: 1009-1022, 1999.

25. Ridley AJ, Paterson HF, Johnston CL, Diekmann D and Hall A: The small GTP-binding protein Rac regulates growth factor-induced membrane ruffling. Cell 70: 401-410, 1992. 